Figure 1 HLA B genotypes profile in our non B 27 autoimmune uveitis patients

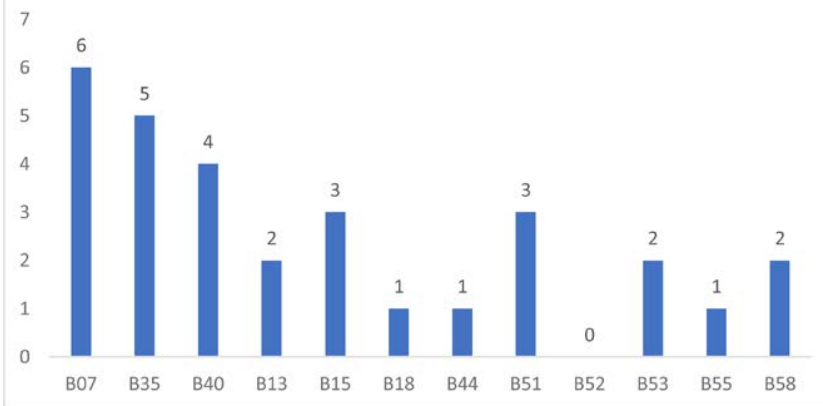

HLA B7 and HLA B35 were the most frequent genotypes (figure 1). Sub group analysis showed that $87.5 \%$ of patients with a component of posterior uveitis were HLA B7 or HLA B35 positive. It was noted none of 5 cases of HLA B35 +ve uveitis needed second line DMARD initiation after a tapering course of systemic steroids. There was no other significant difference in the clinical or treatment parameters noted in the subgroup analysis between the HLA genotypes.

Conclusion: Autoimmune uveitis not associated with HLA B27 tends to have older age of onset, female predominance, more common involvement of posterior uvea and association of vasculitis than HLAB27 autoimmune uveitis. Among non HLA B27 uveitis, HLA B07 and HLA B35 are the most common serotypes in our Asian south Indian group. Therapeutic stratification for a delayed initiation of second line DMARD therapy needs to be studied further particularly in HLA B35 +ve uveitis.

References:

[1] Ninan F, Mathew J, Philip SS, John D, Danda D, John SS. Uveitis of spondyloarthritis in Indian subcontinent: a cross sectional study. Int J Adv Med 2017:4:1441-6

Disclosure of Interests: None declared

DOI: 10.1136/annrheumdis-2020-eular.5140

\section{SAT0539 MUCKLE-WELLS SYNDROME IN RHEUMATOLOGY PRACTICE, FAMILY CASES: FEDERAL CENTER EXPERIENCE.}

S. Salugina $^{1}$, E. Fedorov ${ }^{1}$, K. Elena ${ }^{2}$, E. Zakharova ${ }^{2}$, S. Palshina ${ }^{1}$

${ }^{1}$ V.A.Nasonova Research Institute of Rheumatology, Moscow, Russian Federation; ${ }^{2}$ Research Centre for Medical Genetics, Laboratory of hereditary metabolic diseases, Moscow, Russian Federation

Background: Muckle-Wells syndrome (MWS) is a monogenic autoinflammatory disease caused by a NLRP3 gene mutation. It is the most common variant of cryopyrin-associated periodic syndromes (CAPSs) and can be observed in rheumatology practice. It manifests itself in fever, urticaria-like rash, arthralgias/ arthritides, conjunctivitis/uveitis, sensorineural hearing loss, acute-phase markers (ESR, CRP). The disease's onset usually takes place in infancy. There are examples of family cases. Targeted therapy: interleukin-1 inhibitors (anakinra, canakinumab).

Objectives: to provide characteristics of MWS patients, family cases in the rheumatology practice of the Federal Rheumatology Center in Russia.

Methods: in a 10-year period (2009 to 2019), MWS was diagnosed in 42 outpatient and inpatient patients, among them were 24 children, 18 adults, and 9 family cases. All of them underwent a standard rheumatology examination, including a ECR, CRP, ophthalmologist examination, and an audiogram. A molecular genetic test of the NLRP3 gene was carried out for all patients, the diagnosis was confirmed in all of them.

Results: Out of 18 adult patients aged between 19 and 59 years, women were prevalent (16 to 2), the onset age was 0 to 53 years, in $88,9 \%$ cases the onset took place before a patient was 18 years old. When diagnosed, the disease duration varied from 6 to 46 years. Most patients demonstrated fever, urticarial-like rash, arthralgias/arthritides, which were observed in 16 patients $(88.9 \%)$, conjunctivitides were observed in 15 patients $(83,3 \%)$, sensorineural hearing loss - in 8 patients $(44,4 \%)$, abdominal pain, nausea, vomiting - in 4 patients, headache, dizziness - in 6 patients. There also were rare manifestations, such as: recurrent oral ulcers (8), genital ulcers (3), erythema nodosum (3), sore throat and raids on the tonsils (PFAPA-like phenotype) was observed in 2 patients. In 3 patients manifestations were triggered by cold temperature. All patients had an increased ESR and C-reactive protein concentration. Eight family cases of MWS were identified (in total 26 family members aged between 2.5 and 62 years) with a number of affected in one family ranging from 2 to 6 people of different age ( 8 children, 18 adults, out of which 20 were female, and 6 were male). Most patients had fever
(17), urticarial-like rash (18), conjunctivitides (12), oral ulcers (7), articular syndrome (14), sensorineural hearing loss (5), and 2 patients died of renal insufficiency (probably due to amyloidosis of the kidneys). The heterozygous mutations in NLRP3 have been identified in pts: T348M (3 families), R262W (2 families), A439V (1), V198M (1), Pro294Ser (1). Ten patients received canakinumab for a period of 6 months to 6.5 years, and 5 patients received anakinra before canakinumab. Conclusion: MWS is an orphan autoinflammatory disease, however it sometimes can be observed in rheumatology practice. It is very important to acquire family medical history to identify affected family members and prescribe therapy in a timely manner. IL-1 inhibitors are an effective and safe treatment option for MWS patients. Disclosure of Interests: None declared

DOI: 10.1136/annrheumdis-2020-eular.2167

SAT0540

ONE-YEAR OUTCOMES AFTER RHEUMATIC IMMUNERELATED ADVERSE EVENTS FROM CHECKPOINT INHIBITORS

E. Berard ${ }^{1}$, T. Barnetche ${ }^{1}$, L. Rouxel ${ }^{1}$, C. Dutriaux ${ }^{2}$, L. Dousset ${ }^{2}$, S. Prey ${ }^{2}$, M. Beylot-Barry ${ }^{2}$, J. Seneschal ${ }^{2}$, R. Veillon ${ }^{3}$, C. Vergnenegre ${ }^{3}$, A. Daste ${ }^{4}$, C. Domblides ${ }^{4}$, B. Sionneau ${ }^{4}$, M. Gross-Goupil ${ }^{4}$, A. Ravaud ${ }^{4}$, E. Forcade ${ }^{5}$, T. Schaeverbeke ${ }^{1}$, M. Kostine ${ }^{1} .{ }^{1}$ Bordeaux University Hospital, Rheumatology, Bordeaux, France; ${ }^{2}$ Bordeaux University Hospital, Dermatology, Bordeaux, France; ${ }^{3}$ Bordeaux University Hospital, Pulmonology, Bordeaux, France; ${ }^{4}$ Bordeaux University Hospital, Oncology, Bordeaux, France; ${ }^{5}$ Bordeaux University Hospital, Hematology, Bordeaux, France

Background: Description and initial management of rheumatic immune-related adverse-events (irAEs) from cancer immunotherapies have been reported by several groups but to date, few studies have evaluated the long-term outcomes and management of rheumatic irAEs (1).

Objectives: To describe the long-term management and assess the one-year outcomes of patients who experienced rheumatic immune-related adverse events (irAEs) due to immune checkpoint inhibitors (ICI).

Methods: This was a single-centre prospective observational study including patients referred for musculoskeletal symptoms while treated with $\mathrm{ICl}$. After baseline rheumatological evaluation defining the clinical entity presented, follow-up visits were organised according to the type and severity of irAE. At one year, persistence of irAE, ongoing treatment, as well as cancer outcomes were assessed. Results: 63 patients were included between September 2015 and June 2018 24 patients $(38 \%)$ presented with non-inflammatory musculoskeletal conditions managed with short-term symptomatic treatment and did not require specific follow-up. 39 patients (62\%) experienced inflammatory manifestations, mimicking either rheumatoid arthritis (RA, $n=19$ ), polymyalgia rheumatica (PMR, $n=16$ ), psoriatic arthritis (PsA, $n=3$ ) and one flare of a preexisting axial spondyloarthritis. Overall, 32 patients ( $82 \%$ ) received systemic glucocorticoids, with a median rheumatic dosage of $15 \mathrm{mg} /$ day (range: $5-60 \mathrm{mg} /$ day). None of the patients had to permanently discontinue $\mathrm{ICI}$ therapy for rheumatic irAE. 20 patients $(67 \%)$ were still receiving glucocorticoids at one year, with a median dosage of $5 \mathrm{mg} /$ day (range: 2-20mg/day). Glucocorticoids were more frequently discontinued for patients with RA-like condition (44\%) than PMR-like condition $(23 \%)$, but no other predictive factor of glucocorticoids withdrawal could be identified. At one year, overall survival and progression-free survival were comparable between patients who were still receiving glucocorticoids for rheumatic irAE and patients who have discontinued. Eight patients required csDMARDs.

Conclusion: At one year, a majority of patients required long-term low-dose glucocorticoids for chronic rheumatic irAE, which seems not altering oncological control.

References:

[1] Braaten TJ, Brahmer JR, Forde PM, et al. Immune checkpoint inhibitor-induced inflammatory arthritis persists after immunotherapy cessation. Ann Rheum Dis. 2019 Sep 20.

Disclosure of Interests: None declared

DOI: 10.1136/annrheumdis-2020-eular.4366

\section{SAT0541 THE SEVERITY OF FMF MAY BE ASSOCIATED WITH CO-MORBIDITIES}

M. E. Tezcan ${ }^{1}$, N. Şen ${ }^{1}$, M. Yılmaz ${ }^{2}$, Ö. Volkan ${ }^{1}$, E. Tükel ${ }^{2}$, S. Yilmaz-Oner ${ }^{1}$. ${ }^{1}$ Kartal Dr. Lutfi Kirdar Training and Research Hospital, Rheumatology, Istanbul, Turkey; ${ }^{2}$ Kartal Dr. Lutfi Kirdar Training and Research Hospital, Internal Medicine, Istanbul, Turkey

Background: Familial Mediterranean fever (FMF) is an auto inflammatory disease with recurrent attacks of serositis. Frequent attacks and disease related sequels may be associated with co-morbidities in FMF patients.

Objectives: One of the tools for evaluating the FMF severity is the international severity scoring system for FMF (ISSF) ${ }^{1}$. This score includes disease related sequels, acute phase measurements, attack features and exertional leg pain 